\title{
Using contracting band to improve right ventricle ejection fraction for patients with repaired tetralogy of Fallot: A modeling study using patient-specific CMR-based 2-layer anisotropic models of human right and left ventricles
}

Chun Yang, MS, ${ }^{\mathrm{a}, \mathrm{b}}$ Dalin Tang, PhD, FAHA, ${ }^{\mathrm{a}}$ Tal Geva, MD, ${ }^{\mathrm{c}}$ Rahul Rathod, MD,${ }^{\mathrm{c}}$ Haruo Yamauchi, MD, $\mathrm{PhD},{ }^{\mathrm{d}}$ Vasu Gooty, MD, ${ }^{\mathrm{c}}$ Alexander Tang, ${ }^{\mathrm{c}}$ Glenn Gaudette, PhD, ${ }^{\mathrm{e}}$ Kristen L. Billiar, PhD, ${ }^{\mathrm{e}, \mathrm{f}}$ Mehmet H. Kural, MS, ${ }^{\mathrm{e}}$ and Pedro J. del Nido, $\mathrm{MD}^{\mathrm{d}}$

Objective: Patients with repaired tetralogy of Fallot account for most cases of late-onset right ventricle failure. The current surgical approach, which includes pulmonary valve replacement/insertion, has yielded mixed results. A new surgical option of placing an elastic band in the right ventricle is proposed to improve right ventricular cardiac function as measured by the ejection fraction.

Methods: A total of 20 computational right ventricular/left ventricular/patch/band combination models using cardiac magnetic resonance imaging from a patient with tetralogy of Fallot were constructed to investigate the effect of band material stiffness variations, band length, and active contraction. These models included 4 different band material properties, 3 band length, 3 active contracting band materials, and models with patch and scar replaced by contracting tissue.

Results: Our results indicated that the band insertion, combined with active band contraction and tissue regeneration techniques that restore right ventricular myocardium, has the potential to improve right ventricular ejection fraction by $7.5 \%$ (41.63\% ejection fraction from the best active band model to more than $34.10 \%$ ejection fraction from baseline passive band model) and $4.2 \%$ (41.63\% from the best active band model compared with cardiac magnetic resonance imaging-measured ejection fraction of $37.45 \%$ ).

Conclusions: The cardiac magnetic resonance imaging-based right ventricular/left ventricular/patch/band model provides a proof of concept for using elastic bands to improve right ventricular cardiac function. Band insertion, combined with myocardium regeneration techniques and right ventricular remodeling surgical procedures, has the potential to improve ventricular function in patients with repaired tetralogy of Fallot and other similar forms of right ventricular dysfunction after surgery. Additional investigations using in vitro experiments, animal models, and, finally, patient studies are warranted. (J Thorac Cardiovasc Surg 2013;145:285-93)

Supplemental material is available online.

From the Mathematical Sciences Department, ${ }^{\mathrm{a}}$ Worcester Polytechnic Institute, Worcester, Mass; Beijing Normal University School of Mathematical Sciences, ${ }^{b}$ Key Laboratory of Mathematics and Complex Systems, Ministry of Education, Beijing, China; Department of Cardiology, ${ }^{\mathrm{C}}$ Children's Hospital Boston and Department of Pediatrics, Harvard Medical School, Boston, Mass; Department of Cardiac Surgery, ${ }^{\mathrm{d}}$ Children's Hospital Boston and Department of Surgery, Harvard Medical School, Boston, Mass; Department of Biomedical Engineering, ${ }^{,}$Worcester Polytechnic Institute, Worcester, Mass; and Department of Surgery, ${ }^{\mathrm{f}}$ University of Massachusetts Medical School, Worcester, Mass.

This research was supported in part by National Institutes of Health grant 1R01-HL 089269 (to P.J.d.N., D.T., T.G.), National Institutes of Health grant HL63095 (primary investigator, P.J.d.N.), and National Institutes of Health grant NHLBI 5P50HL074734 (primary investigator, T.G.; co-investigator: P.J.d.N.). C.Y. research supported in part by National Sciences Foundation of China grant 11171030 and the Fundamental Research Funds for the Central Universities.

Disclosures: Authors have nothing to disclose with regard to commercial support.

Received for publication Dec 27, 2011; revisions received Feb 9, 2012; accepted for publication March 12, 2012; available ahead of print April 9, 2012.

Address for reprints: Dalin Tang, PhD, FAHA, Mathematical Sciences Department,

Worcester Polytechnic Institute, Worcester, MA 01609 (E-mail: dtang@ wpi.edu). $0022-5223 / \$ 36.00$

Copyright $\odot 2013$ by The American Association for Thoracic Surgery

doi:10.1016/j.jtcvs.2012.03.009
Patients with repaired Tetralogy of Fallot (TOF), a congenital heart defect that includes a ventricular septal defect and severe right ventricular (RV) outflow obstruction, account for most cases of late-onset RV failure. The current surgical approach, which includes pulmonary valve replacement/ insertion (PVR), has yielded mixed results, with many patients not recovering RV function after PVR with or without concomitant $\mathrm{RV}$ remodeling surgery. ${ }^{1-3}$ Recent advances in computational modeling, methods, and computer technology have made it possible for computer-simulated procedures to be used in the clinical decision-making process to replace empirical and often risky clinical experimentation to examine the efficiency and suitability of various reconstructive procedures in diseased hearts. ${ }^{4-16}$ Peskin $^{4,5}$ pioneered the heart modeling effort with his celebrated immersed boundary method. More recently, McCulloch and colleagues, ${ }^{6}$ Kerckhoffs and colleagues, ${ }^{7}$ Hunter and colleagues, ${ }^{8}$ Nash and Hunter, ${ }^{9}$ Costa and colleagues, ${ }^{10}$ Guccione and colleagues, ${ }^{11}$ Axel,,${ }^{12}$ and Saber and colleageus, ${ }^{13}$ as well as many other investigators, have made great contributions to passive and active ventricle modeling, including 


$$
\begin{aligned}
& \text { Abbreviations and Acronyms } \\
& \text { ABM }=\text { active band model } \\
& \text { 3D }=3 \text {-dimensional } \\
& \text { CMR }=\text { cardiac magnetic resonance } \\
& \text { LV }=\text { left ventricular } \\
& \text { MRI }=\text { magnetic resonance imaging } \\
& \text { PBM }=\text { passive band model } \\
& \text { PVR }=\text { pulmonary valve replacement/insertion } \\
& \text { RV }=\text { right ventricular } \\
& \text { TOF }=\text { tetralogy of Fallot }
\end{aligned}
$$

the Physiome Project and the continuity package. Early magnetic resonance imaging (MRI)-based ventricle models were introduced by Axel ${ }^{12}$ and Saber and colleagues ${ }^{13}$ for mechanical analysis and investigation. In our previous reports, patient-specific cardiac magnetic resonance (CMR) image-based computational RV and left ventricular (LV) models with fluid-structure interactions were introduced to assess the outcomes of various RV reconstruction techniques with different scar tissue trimming and patch sizes. ${ }^{14-16} \mathrm{~A}$ recent review can be found in the report by Tang and colleagues. ${ }^{14}$

For patients with TOF who need PVR surgery, surgical intervention such as scar tissue resection and anterior wall $\mathrm{RV}$ remodeling has been proposed to improve $\mathrm{RV}$ function recovery. ${ }^{17} \mathrm{RV}$ remodeling aims mainly to reduce the RV volume by removing or reducing the noncontracting tissue (scar and patch) in the outflow area of the RV (Figure 1). In our clinical trial, 64 patients who underwent RV outflow tract procedures in early childhood had more than or equal to moderate pulmonary regurgitation and fulfilled the defined criteria for PVR were randomly assigned to undergo either PVR alone $(\mathrm{n}=34)$ or PVR with surgical RV remodeling $(n=30)$. However, no significant difference was observed in the primary outcome (change in RV ejection fraction $[\mathrm{EF}],-2 \% \pm 7 \%$ in the PVR-alone group vs $-1 \% \pm 7 \%$ in the PVR with RV remodeling group; $P=.38$ ) or in any of the secondary outcomes at 6 months postoperatively. ${ }^{18}$ We are currently attempting to identify the factors that might help us to identify those patients who would benefit from the PVR remodeling procedures using a subanalysis. ${ }^{16}$

In the present report, in our continuous effort to find new surgical options for better RV function recovery, we have used our mathematical models of the late postoperative RV to evaluate the effect of novel interventions. To this end, we have introduced the concept of an elastic band with varying elastic properties and the capacity for active force generation similar to the normal myocardium. This band concept was added to our previous patientspecific MRI-based RV/LV/patch models to investigate its effect and potential improvement on RV cardiac function and mechanical performance. The band models included an elastic band connecting the RV anterior free wall and the septum; 2-layer ventricle myocardial wall construction with realistic epicardial and endocardial fiber orientation, replacing the previous RV outflow nonmyocardium patch; anisotropic material properties for both RV and LV tissue; a patch/scar area with different materials; and the LV as support to the RV structure. Because we were mainly concerned with whether addition of the band would lead to an improved RVEF, structure-only models were used, instead of our previous models, which included fluid-structure interactions. A total of 20 models based on MRI studies obtained in patients being evaluated for surgery to treat RV dilation and dysfunction late after TOF repair, were constructed, including 4 different band material properties, 3 band lengths, 1 passive and 3 active contracting band materials, and models with patch and scar all replaced by contracting myocardium. The 3-dimensional (3D) CMR-based RV/LV/patch/band models were solved to obtain 3D ventricular deformation and stress/strain distributions for accurate assessment of $\mathrm{RV}$ cardiac function and mechanical conditions. The computational models were validated using the CMR data first and then used to assess the effect of different band approaches, with the ultimate goal of improving recovery of RV function after surgery.

\section{DATA ACQUISITION, MODELS AND METHODS Data Acquisition, 3D Geometry Reconstruction, and Band Location}

CMR studies were performed by 1 of us (T.G.) to acquire patient-specific ventricular geometry for patients requiring RV remodeling and PVR surgery before and after surgery (Figure 2, A). The right and left ventricles were imaged using electrocardiography-gated, breath-hold, steady-state, free precession cine MRI in the ventricular short axis (12-14 equidistant slices covering the ventricles from the base to apex; slice thickness, $6-8 \mathrm{~mm}$; interslice gap, $0-2 \mathrm{~mm} ; 30$ frames per cardiac cycle). The valve and patch positions were determined with cine MRI, flow data, and delayed enhancement CMR to delineate the location and extent of scar or patch. The CMR findings were subsequently confirmed by intraoperative observation (P. J. d. N.). 3D RV/LV geometry and computational mesh were constructed according to the procedures described by Tang and colleagues. ${ }^{14-16}$ Figure 2 shows the preoperative CMR images from the patient with repaired TOF and severe RV dilation, segmented contour plots, stacked contours showing the location and orientation of the hypothetic elastic band, and 3D reconstruction RV/LV geometry with a front view showing the patch, scar, band, and RV outflow track. The location of the band was carefully chosen to help the right ventricle to contract in the present model. 


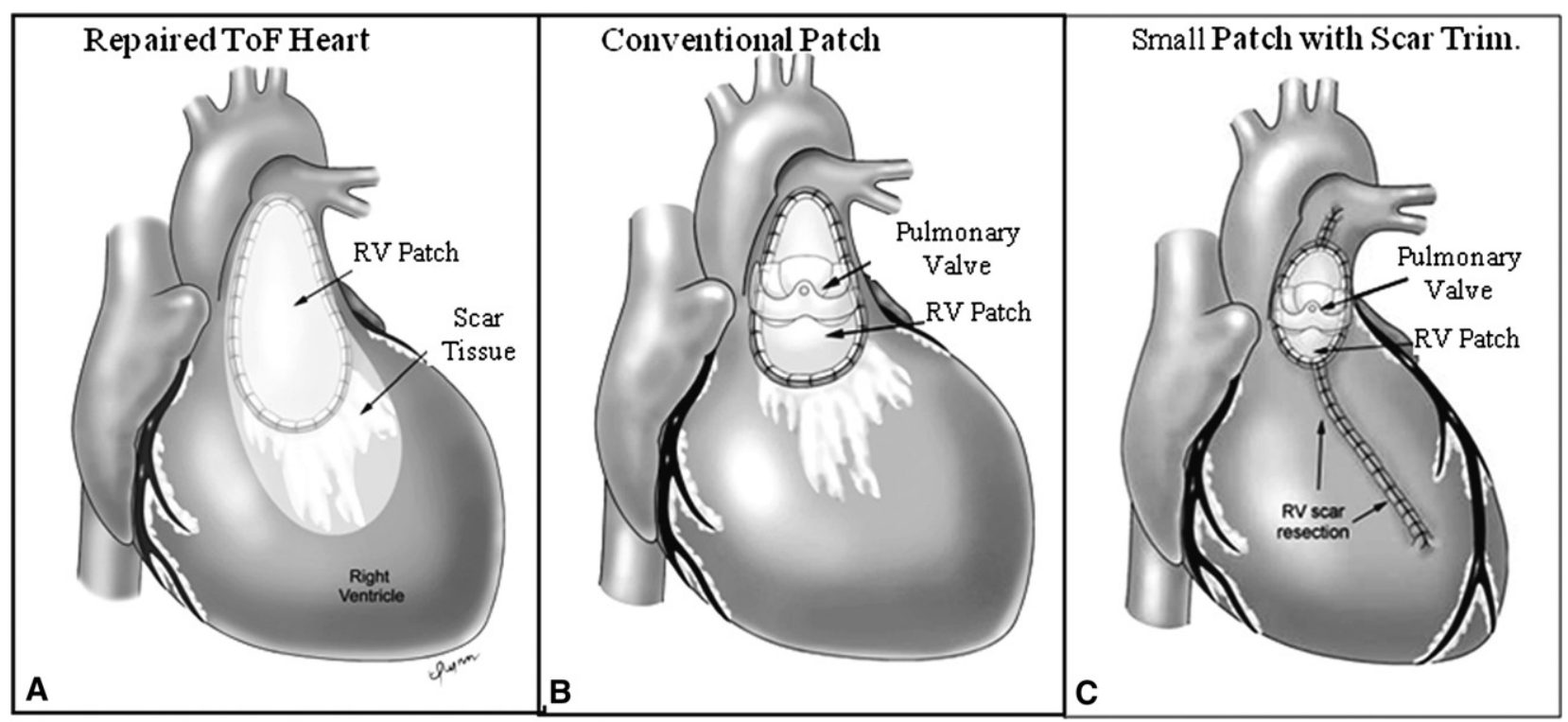

FIGURE 1. Hypothesis that smaller patch and removal of RV scar might lead to improved surgical outcome after surgery. A, Repaired tetralogy of Fallot $(T o F)$ heart with right ventricular $(R V)$ outflow patch and scar on anterior RV wall. B, Right ventricular after pulmonary valve insertion $(P V R)$ and reduction of RV outflow patch (current practice). C, Right ventricle after pulmonary valve insertion $(P V R)$, reduction of RV outflow patch, and removal of RV scar (RV remodeling, proposed new surgical procedure).

\section{Anisotropic Material Models for RV Tissue and Band, Fiber Orientation, and 2-Layer Model Construction}

The governing equations for all material models were:

$$
\begin{gathered}
\rho v_{\mathrm{i}, \mathrm{tt}}=\sigma_{\mathrm{ij}, \mathrm{j}}, \quad \mathrm{i}, \mathrm{j}=1,2,3 \text { sum over } \mathrm{j}, \\
\varepsilon_{i j}=\left(v_{\mathrm{i}, \mathrm{j}}+v_{\mathrm{j}, \mathrm{i}}+v_{\alpha, \mathrm{i}} v_{\alpha, \mathrm{j}}\right) / 2, \mathrm{i}, \mathrm{j}, \alpha=1,2,3,
\end{gathered}
$$

where $\sigma$ is the stress tensor, $\varepsilon$ is the strain tensor, $\mathrm{v}$ is displacement, $f \bullet, j$ is the derivative of $f$ with respect to the jth variable, and $\rho$ is the material density. The normal stress was assumed to be 0 on the outer RV/LV surface and equal to the normal stress imposed by fluid pressure on the inner RV/LV surfaces. Structure-only RV/LV models were used to save model construction effort and computing time. They were adequate for our purpose in the present report to obtain the RV volume and EFs for ventricle cardiac function assessment.

The RV and LV materials were assumed to be hyperelastic, anisotropic, nearly incompressible, and homogeneous. The band, patch, and scar materials were assumed to be hyperelastic, isotropic, nearly incompressible, and homogeneous. The nonlinear Mooney-Rivlin model was used to describe the nonlinear anisotropic and isotropic material properties. The strain energy function for the isotropic modified Mooney-Rivlin model is given by the following equation $^{14,19}$ :

$$
\mathrm{W}=\mathrm{c} 1\left(\mathrm{I}_{1}-3\right)+\mathrm{c} 2\left(\mathrm{I}_{2}-3\right)+\mathrm{D}_{1}\left[\exp \left(\mathrm{D}_{2}\left(\mathrm{I}_{1}-3\right)\right)-1\right],
$$

where $I_{1}$ and $I_{2}$ are the first and second strain invariants given by,

$$
\mathrm{I}_{1}=\sum C_{i i}, \mathrm{I}_{2}=1 / 2\left[\mathrm{I}_{1}^{2}-\mathrm{C}_{\mathrm{ij}} \mathrm{C}_{\mathrm{ij}}\right],
$$

$\mathrm{C}=\left[\mathrm{C}_{\mathrm{ij}}\right]=\mathrm{X}^{\mathrm{T}} \mathrm{X}$ is the right Cauchy-Green deformation tensor, $\mathrm{X}=\left[\mathrm{X}_{i j}\right]=\left[\partial \mathrm{x}_{\mathrm{i}} / \partial \mathrm{a}_{\mathrm{j}}\right],\left(\mathrm{x}_{\mathrm{i}}\right)$ is the current position, $\left(a_{i}\right)$ is the original position, $c_{i}$ and $D_{i}$ are material parameters chosen to match experimental measurements..$^{14,20,21}$ The strain energy function for the anisotropic modified Mooney-Rivlin model was obtained by adding an additional anisotropic term in Equation $3^{19,22}$ :

$$
\begin{aligned}
\mathrm{W}= & \mathrm{c}_{1}\left(\mathrm{I}_{1}-3\right)+\mathrm{c}_{2}\left(\mathrm{I}_{2}-3\right)+\mathrm{D}_{1}\left[\exp \left(\mathrm{D}_{2}\left(\mathrm{I}_{1}-3\right)\right)-1\right] \\
& +\mathrm{K}_{1} /\left(2 \mathrm{~K}_{2}\right) \exp \left[\mathrm{K}_{2}\left(\mathrm{I}_{4}-1\right)^{2}-1\right]
\end{aligned}
$$

where $I_{4}=\mathrm{C}_{\mathrm{ij}}\left(\mathbf{n}_{\mathrm{f}}\right)_{\mathrm{i}}\left(\mathbf{n}_{\mathrm{f}}\right)_{\mathrm{j}}, \mathrm{C}_{\mathrm{ij}}$ is the Cauchy-Green deformation tensor, $n_{f}$ is the fiber direction, and $K_{1}$ and $K_{2}$ are material constants. ${ }^{19}$ A 2-step least-squares method was used to determine the parameter values in Equation 5 to fit the experimental data given in McCulloch and colleagues. ${ }^{6}$ Step 1: with $\mathrm{D}_{2}$ and $\mathrm{K}_{2}$ fixed, the least square approximation technique was used to obtain $\mathrm{C}_{1}, \mathrm{D}_{1}$, and $\mathrm{K}_{1}$ (all dependent on $\mathrm{D}_{2}$ and $\mathrm{K}_{2}$ ) so that the stress-strain curves in the fiber and circumferential directions derived from Equation 5 have minimum error (best match) with experimental data. Step 2: let $\mathrm{D}_{2}$ and $\mathrm{K}_{2}$ change from -100 to 100 , we perform Step 1 to find the corresponding $\mathrm{C}_{1}, \mathrm{D}_{1}$, and $\mathrm{K}_{1}$ values, and the fitting error for all $\left(\mathrm{D}_{2}, \mathrm{~K}_{2}\right)$ combinations with an initial increment of 10 . Optimal $\left(\mathrm{D}_{2}, \mathrm{~K}_{2}\right)$ and the associated 
Pre-Operation CMR Images from a Patient, End of Systole

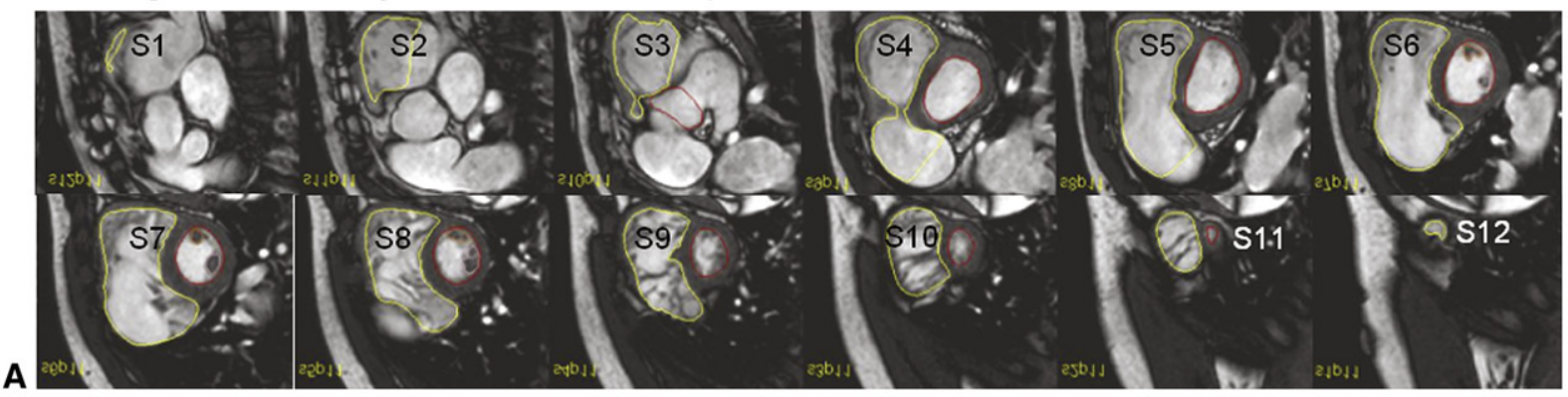

Segmented Contours of RV-LV for Model Construction

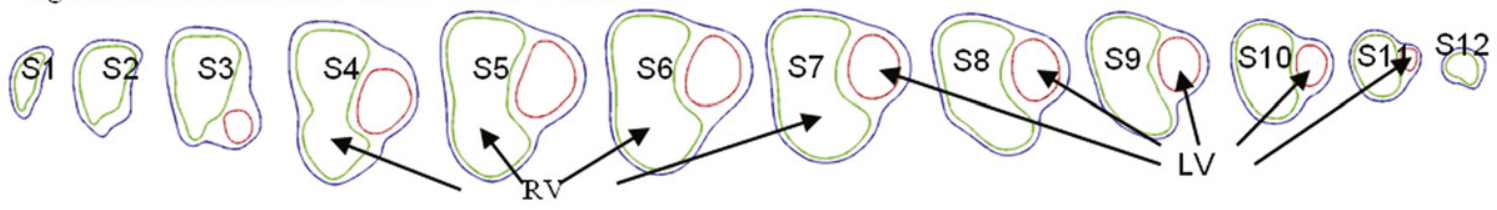

B

Re-constructed RV/LV geometry with patch and scar; location of the band.

C
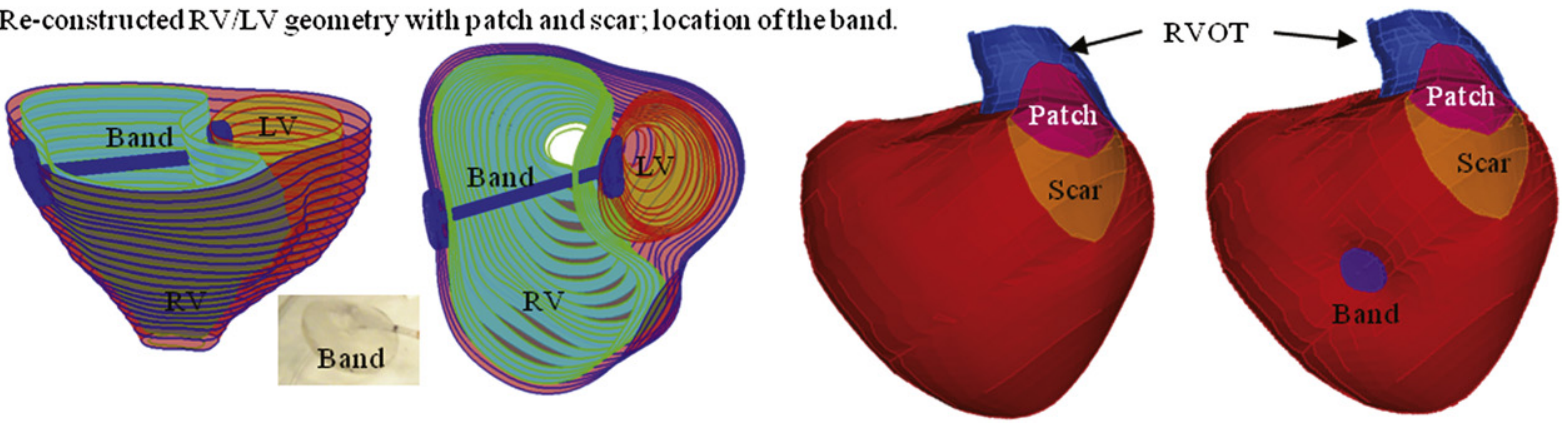

FIGURE 2. A-C, Cardiac magnetic resonance (CMR) images of patient, segmented contours, and reconstructed 3-dimensional (3D) geometry with and without a band. $R V-L V$, Right ventricle-left ventricle; $R V O T$, right ventricular outflow track.

$\mathrm{C}_{1}, \mathrm{D}_{1}, \mathrm{~K}_{1}$ values are determined by choosing the pair corresponding to a minimum error. The searching increment for $\left(D_{2}, K_{2}\right)$ starts from 10 for $[-100,100]$ and then refines to 1 and 0.1 when the search domain is reduced. Although the procedure involves a huge number of calculations, it is fully automatic and can be used to find the best fit for any measured experimental data. Choosing $\mathrm{c}_{1}=0.351$ $\mathrm{KPa}, \mathrm{c}_{2}=0, \mathrm{D}_{1}=0.0633 \mathrm{KPa}, \mathrm{D}_{2}=5.3, \mathrm{~K}_{1}=1.913$ $\mathrm{KPa}, \mathrm{K}_{2}=6.00$, it was shown ${ }^{14,15}$ that the stress-strain curves derived from Equation 5 agreed very well with the stress-strain curves from the dog model given in the report by McCulloch and colleagues. ${ }^{6}$ The parameter values were then adjusted to fit CMR-measured RV volume data. The stress-stretch curves and parameter values of the RV/LV tissues, patch, scar and band are given in Figure E1. Imposed $\mathrm{RV}$ pressure conditions and computational RV volume data from our baseline no-band model are given in Figure E1, $C$ and $D$. Good agreement between the computational and CMR-measured volume data was found (error $<2 \%$ ).

For the elastic band model, uniaxial mechanical testing was performed using 3 sample bands made of Tecoflex EG-80A (Lubzizol Advanced Materials, Cleveland, Ohio) to obtain the material parameter values for our band material. The stress-stretch curves from Mooney-Rivlin model fitting mechanical engineering test data of the Tecoflex band and 5 other materials are shown in Figure E1, $B$.

Three active band contraction models (with $10 \%, 15 \%$, and $20 \%$ band zero-stress length reduction) were evaluated to measure the improvements, if any, in RVEF. An "actively contracting band" should be made of material that is able to contract the same as normal myocardium. The present report is mainly to demonstrate the potential benefit of actively contracting bands if such materials could be made available. Active band contraction was achieved using an iterative procedure and coupling a separate simple band-only model to the whole RV/LV/patch/band model. This was done to adjust the band zero-stress length (contraction at the start of systole and relaxation at the start of diastole); recalculate the band displacement, stress, and strain using the band-only model; and assign the displacement, stress, and strain values to the band in the right ventricle as the start for the whole model with a new zero-stress band length.

Because patient-specific fiber orientation data was not available in practice, we chose to construct a 2-layer RV/ LV model and set the fiber orientation angles using the fiber angles given by Hunter and colleagues ${ }^{8}$ (Figure 3). Fiber 
Pig Model,

Epicardium

A

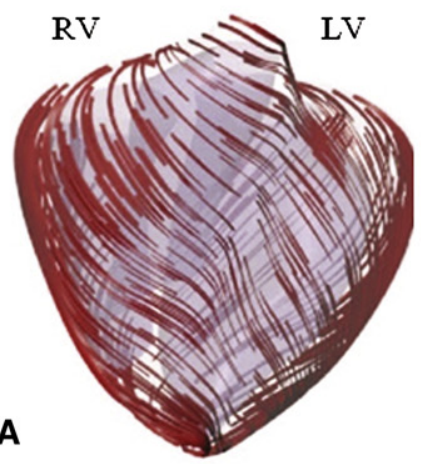

Patient Model,

Epicardium

E

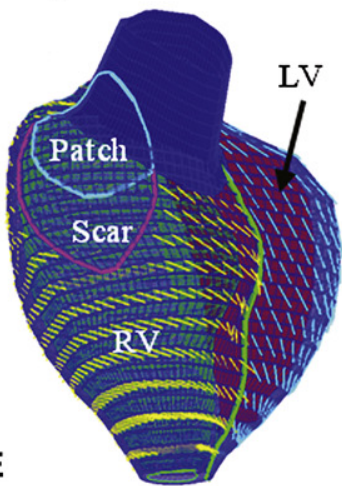

Pig Model, Endocardium

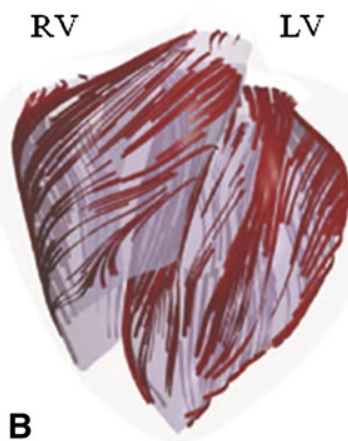

Patient Model,

Endocardium.

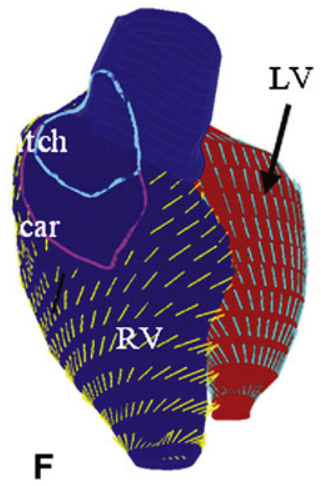

Human Heart, Front View
Human Heart, Back View

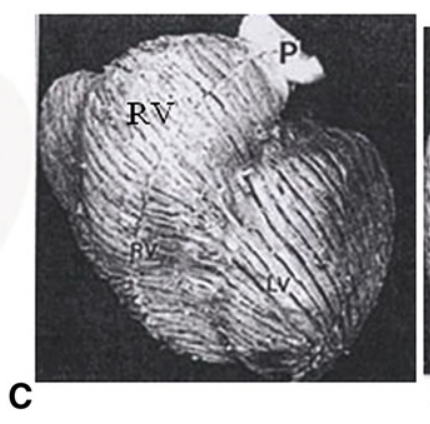

Two-layer Construction

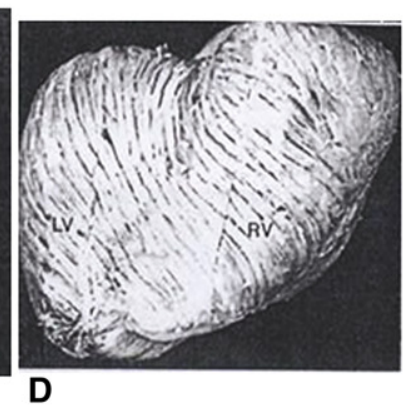

D
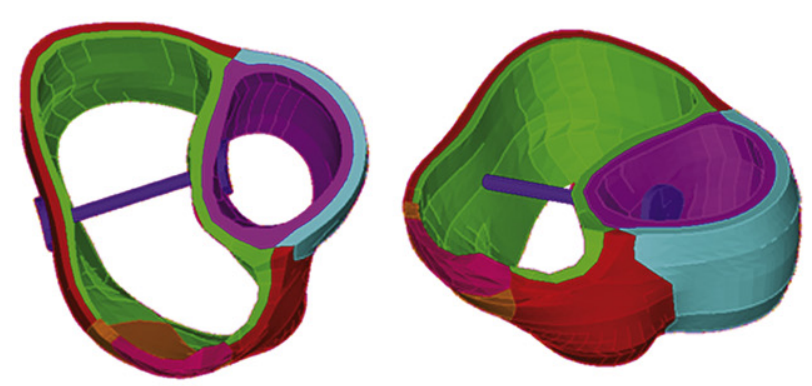

G

FIGURE 3. Fiber orientation and 2-layer model construction. Fiber orientations from pig model on (A) epicardium and (B) endocardial surface. Left ventricular $(L V)$ fiber orientation was approximately $-60^{\circ}$ (relative to circumferential direction) at the epicardium and $+80^{\circ}$ at the endocardium. $\mathrm{C}$, Human ventricular fiber orientation from a patient: $\mathrm{C}$, font view; D, back view. $\mathrm{E}$ and $\mathrm{F}$, Fiber orientation from our proposed right ventricular $(R V) /$ left ventricular $(L V)$ model based on patient-specific RV/LV morphologies. RV fiber orientation was set at $-45^{\circ}$ at the epicardium and $+40^{\circ}$ at the endocardium. Angles can be adjusted to fit patient-specific data. G, Two-layer model construction illustration using 2 slices.

orientation can be adjusted when patient-specific data become available. ${ }^{23}$ Figure 3 shows the ventricular fiber orientations on the epicardium and endocardium layers from a human and pig heart and how the 2-layer RV/LV model was constructed. ${ }^{8,14,23}$

\section{Mesh Generation: Preshrink Process and Geometry- Fitting Technique for Patient-Specific CMR-Based Models}

Under the in vivo condition, the ventricles were pressurized, and the zero-stress ventricular geometries were not known. In our model construction process, a preshrink process was applied to the in vivo end-systolic ventricular geometries to generate the starting shape (zero ventricle pressure) for the computational simulation. The initial shrinkage for the inner ventricular surface was $2 \%$ to $3 \%$ and end-systolic pressure was applied so that the ventricles would regain its in vivo morphology. The outer surface of the ventricular shrinkage was determined by conservation of mass such that the total ventricular wall mass was conserved. Without this shrinking process, the actual computing domain would be greater than the actual ventricle owing to the initial expansion when pressure was applied.

Because ventricles have complex irregular geometries with patch and scar tissue component inclusions that are challenging for mesh generation, a geometry-fitting mesh generation technique was developed to generate mesh for our models. Figure 3, $G$ gives an illustration of RV/LV geometry between 2 slices. Each slice was first divided into geometry-fitting areas (termed "surfaces" in ADINA; ADINA R\&D, Watertown, Mass). The neighboring slices were stacked to form volumes. Using this technique, the 3D RV/LV domain was divided into many small "volumes" to curve-fit the irregular plaque geometry with patch as an inclusion. For the baseline $\mathrm{RV} / \mathrm{LV} /$ patch model constructed in the present study, the ADINA model (ADINA R\&D) had 1759 small volumes, 30,273 nodes, and 26,276 elements. Mesh analysis was performed by decreasing the mesh size by $10 \%$ (in each dimension) until the solution differences were less than $2 \%$. The mesh was then chosen for our simulations. 


\section{Model List, Solution Methods, and Simulation Procedures}

Twelve models with 4 different material stiffnesses (1 stiff, 0.5 stiff, 0.25 stiff, and 0.05 stiff; parameter values given in Figure E1) and 3 different band length (100\%, $90 \%$ and $80 \%$ of the original baseline model length) were constructed to evaluate the effect of band material stiffness and length on RV cardiac function commonly measured by its stroke volume (SV) and EF, defined as follows:

$$
\begin{aligned}
& S V= \text { RV End Diastolic Volume (RVEDV) } \\
&- \text { RV End Systolic Volume }(\text { RVESV }) ; \\
& \text { EF }=[\text { RVEDV }- \text { RVESV }] / \text { RVEDV }
\end{aligned}
$$

Furthermore, active contraction band models were made by allowing the band to contract $10 \%, 15 \%$, and $20 \%$ to improve the EF values. The full RV/LV/patch/band models were solved by ADINA (ADINA R\&D) using unstructured finite elements and the Newton-Raphson iteration method. ${ }^{19}$ Stress/strain distributions and RVEF were calculated to seek the optimal surgical design for potential RVEF improvement.

\section{RESULTS}

For the patient under consideration, the RVEF from our baseline no-band model was $37.48 \%$, in agreement with the EF data (37.45\%) from direct CMR measurement. A total of 20 models were made from the baseline model to investigate the effect of material and band length variations, active band contraction, and use of viable tissues to replace the patch and scar.

\section{Effect of Band Material Stiffness and Length on RVEF}

Table 1 presents a summary of RVEF from 12 models, with 4 stiffness and 3 band length variations. We started from a fixed stiffness, which was given a value of " 1 " and reduced it incrementally to a stiffness of 0.05 (Table 1), close to the stiffness of the Tecoflex material (Lubzizol Advanced Materials, Cleveland, Ohio). The band was made softer with the hope that softer material would have more passive elastic contractions and would lead to better EF. Our results indicated that the addition of a passive elastic band led to about a 5\% EF reduction for all 12 models considered. A softer band provided slightly better EF (EF was $1.5 \%$ higher). The band length had very little effect on the EF.

\section{Effect of Active Band Contraction on RVEF}

Because passive band materials led to lower EFs, 3 active contractions (band zero-stress length reduced by $10 \%$, $15 \%$, and $20 \%$ ) were added to the band, seeking possible RVEF improvement. Table 2 lists the EF values from the

\begin{tabular}{|c|c|c|c|c|c|}
\hline \multirow[b]{2}{*}{ Model } & \multirow[b]{2}{*}{$\begin{array}{c}\text { Band } \\
\text { stiffness }\end{array}$} & \multirow[b]{2}{*}{$\begin{array}{c}\text { Band } \\
\text { length }(\%)\end{array}$} & \multicolumn{2}{|c|}{ RV volume (mL) } & \multirow[b]{2}{*}{ EF $(\%)$} \\
\hline & & & $\begin{array}{l}\text { End of } \\
\text { systole }\end{array}$ & $\begin{array}{c}\text { End of } \\
\text { diastole }\end{array}$ & \\
\hline CMR data & NA & NA & 254.5 & 406.9 & 37.45 \\
\hline Base model & NA & No band & 252.5 & 403.8 & 37.48 \\
\hline PBM1 & 1 & 100 & 245.9 & 363.4 & 32.33 \\
\hline PBM2 & 0.5 & 100 & 245.9 & 364.1 & 32.47 \\
\hline PBM3 & 0.25 & 100 & 246.0 & 365.6 & 32.71 \\
\hline PBM4 & 0.05 & 100 & 246.4 & 373.0 & 33.94 \\
\hline PBM5 & 1 & 90 & 240.3 & 353.8 & 32.08 \\
\hline PBM6 & 0.5 & 90 & 240.3 & 354.7 & 32.23 \\
\hline PBM7 & 0.25 & 90 & 240.4 & 356.1 & 32.49 \\
\hline PBM8 & 0.05 & 90 & 240.8 & 363.8 & 33.80 \\
\hline PBM9 & 1 & 80 & 234.3 & 344.0 & 31.87 \\
\hline PBM10 & 0.5 & 80 & 234.4 & 344.7 & 32.02 \\
\hline PBM11 & 0.25 & 80 & 234.4 & 346.2 & 32.28 \\
\hline PBM12 & 0.05 & 80 & 234.9 & 354.1 & 33.67 \\
\hline
\end{tabular}

TABLE 1. Summary of right ventricular ejection fractions from 12 models with 4 stiffness and 3 band length variations

Summary of RV ejection fractions from 12 models with 4 stiffness and 3 band length variations; a softer and longer band provided slightly better EF. EF rates from all passive band models was $5 \%$ lower than that with no-band model. Mooney Rivlin model parameters for 4 band stiffness variations: 1 stiff, $\mathrm{c}_{1}=12,000 \mathrm{kPa}, \mathrm{D}_{1}=6000 \mathrm{kPa}$, $\mathrm{D}_{2}=1.4 ; 0.5$ stiff, $\mathrm{c}_{1}=6000 \mathrm{kPa}, \mathrm{D}_{1}=3000 \mathrm{kPa}, \mathrm{D}_{2}=1.4 ; 0.25$ stiff, $\mathrm{c}_{1}=3000$ $\mathrm{kPa}, \mathrm{D}_{1}=1500 \mathrm{kPa}, \mathrm{D}_{2}=1.4 ; 0.05$ stiff, $\mathrm{c}_{1}=600 \mathrm{kPa}, \mathrm{D}_{1}=300 \mathrm{kPa}, \mathrm{D}_{2}=1.4$. Band no-load length. $100 \%-4.18 \mathrm{~cm}$ (distance between 2 locations on ventricular wall from no-band model where band would be placed); $90 \%$ band length, 3.76 $\mathrm{cm} ; 80 \%$ band length, $3.34 \mathrm{~cm}$. $R V$, Right ventricular; $E F$, ejection fraction; $C M R$, cardiac magnetic resonance; $N A$, not applicable; $P B M$, passive band model.

3 active band models (ABM1-ABM3). The EF for the RV with patch and scar improved from $34.10 \%$ (passive band model $[\mathrm{PBM}] 13$, the corresponding passive band model) to $37.43 \%, 39.39 \%$, and $39.92 \%$ for the active band model (ABM)1 ABM2, and ABM3, respectively. The EF improvement from PBM13 to ABM3 was 5.62\%. When the patch and scar were replaced by "normal RV tissue" (ie, the scar area was replaced by myocardial tissue with normal contractile properties), the EF values from the 3 active band models (ABM4-ABM6) improved from 35.59\% (PBM14, the corresponding passive band model) to $39.54 \%, 40.99 \%$, and $41.63 \%$, respectively. The EF improvement from PBM14 to ABM6 was $6.04 \%$.

\section{Stress/Strain Distributions Have Complex Patterns in the Ventricle}

Figure E2 shows the inner RV surface viewed from 1 cut plane, and the corresponding maximum principal stress $\left(\right.$ Stress- $\left.\mathrm{P}_{1}\right)$ and strain (Strain- $\left.\mathrm{P}_{1}\right)$ plots from 3 selected models: a baseline model with patch and scar, no band; a passive band model with patch and scar; and a passive band model with patch and scar replaced by normal tissue. Some mesh lines were kept to better show the 3D surface. Clearly, the RV geometry, curvature, material properties, patch, scar, and band all have considerable contributions to the RV stress/strain distributions. A large curvature 
TABLE 2. Summary of RV ejection fractions from 6 active band models compared with corresponding passive band models

\begin{tabular}{|c|c|c|c|c|}
\hline Model & $\begin{array}{c}\text { Band active } \\
\text { contraction }(\%)\end{array}$ & $\begin{array}{c}\text { End of systole } \\
\text { RV volume (mL) }\end{array}$ & $\begin{array}{l}\text { End of diastole } \\
\text { RV volume (mL) }\end{array}$ & EF $(\%)$ \\
\hline \multicolumn{5}{|l|}{ Patch and scar } \\
\hline Base model & No band & 252.5 & 403.8 & 37.48 \\
\hline PBM13 & Passive & 246.4 & 373.9 & 34.10 \\
\hline ABM1 & 10 & 246.1 & 393.3 & 37.43 \\
\hline ABM2 & 15 & 237.9 & 392.6 & 39.39 \\
\hline ABM3 & 20 & 234.1 & 389.6 & 39.92 \\
\hline \multicolumn{5}{|c|}{ Patch and scar replaced by normal tissue } \\
\hline PBM14 & Passive & 247.1 & 383.7 & 35.59 \\
\hline ABM4 & 10 & 246.7 & 408.1 & 39.54 \\
\hline ABM5 & 15 & 238.6 & 404.4 & 40.99 \\
\hline ABM6 & 20 & 234.7 & 402.1 & 41.63 \\
\hline
\end{tabular}

caused noticeable stress/strain concentrations in all 3 models. High stress/strain concentrations were also found where the band connected to RV. Replacing patch and scar by normal tissue led to smoother stress/strain distributions. This could become possible if and when viable myocardium tissue could be generated using tissue engineering techniques under development. ${ }^{24,25}$

\section{DISCUSSION}

\section{Motivation to Develop Band Model and Clinical Potential}

With the rapidly increasing number of late survivors of TOF repair, the surgical treatment of patients with RV dysfunction has become a major clinical challenge. The wide variability in clinical status, regurgitation at both tricuspid and pulmonary valves, extent of RV dilation, scarring, and dysfunction at presentation has resulted in disparate surgical results with pulmonary valve insertion alone. ${ }^{3}$ del Nido $^{17}$ and Geva and colleagues ${ }^{18}$ have proposed aggressive scar tissue removal and RV volume remodeling as a method to improve RV function after PVR surgery. However, in a randomized prospective clinical trial, this aggressive approach did not result in measurable improvement in RVEF or RV end-diastolic volume compared with pulmonary valve insertion and RV outflow track patch reduction alone. The current proposed band approach is another approach to assist the ventricle to contract and improve its EF. If an active contracting material or actuator could be used for the band material, the band might be able to augment the contraction of the right ventricle. Proulx and colleagues ${ }^{26}$ described a band made from fibrin that could be seeded with mesenchymal stem cells and stitched through a collagen gel. Seeding these fibrin bands with contractile cells, possibly derived from stem cells, might provide an actively contracting band. Also, recent work has suggested that contractile cells can be generated to replace scarred myocardium, providing a contracting scaffold. ${ }^{24,25}$ Combined with the tissue regeneration techniques that restore RV myocardium, the combined scar tissue replacement plus active band approach has the potential to improve the $\mathrm{EF}$ by $7.5 \%$ ( $41.63 \%$ from best $\mathrm{ABM}$ [ABM6] vs $34.10 \%$ from PBM13) and $4.2 \%$ (41.63\% from ABM6 vs $37.45 \%$ from the CMR EF data). The percentage of increase in $\mathrm{EF}$ is significant, representing a $22 \%$ relative improvement over the observed $\mathrm{EF}$ of $34 \%$. The effect on functional status would depend on a number of factors, including LV function. However, a 7\% increase in the RVEF compares favorably with recently published drug trials to treat heart failure in which an improvement in LVEF of 3\% to $4 \%$ resulted in significant improvement in functional capacity. ${ }^{27}$

The computational simulations (virtual surgery) provided a proof of concept for additional investigations using in vitro experiments, animal models, and, finally, patient studies. Computational models are noninvasive and can be used to supplement/replace empirical and often risky clinical experimentation or even guide the design of new clinical trials to examine the efficiency and suitability of various reconstructive procedures in diseased hearts.

\section{Passive RV Band and Tricuspid Regurgitation}

Similar passive ventricular restriction approaches to that we have proposed have been evaluated and implanted in patients with left ventricular dilation and functional mitral regurgitation. An example of this approach is the Coapsys device (Myocor, Maple Grove, Minn). Because the Coapsys device demonstrated effectiveness in reducing not only functional mitral regurgitation but also short-axis LV dimensions ${ }^{28}$ the proposed RV band approach can also be expected to improve RV dimensions and might even be effective in treating the functional tricuspid regurgitation seen in these patients. Considering that annular and ventricular 
dilation contribute to tricuspid regurgitation, the passive band might be effective in reducing regurgitation, although the passive band had little effect on EF in the present study.

\section{Mechanical Analysis and Its Relevance to Ventricular Remodeling, Myocardial Regeneration, and Surgical Design}

It is well known that mechanical forces play an important role in biologic processes. Peskin, ${ }^{4,5}$ Hunter and colleagues, ${ }^{8}$ McCulloch and colleagues, ${ }^{6}$ Guccione and colleagues, ${ }^{11}$ Costa and colleagues, ${ }^{10}$ Kerckhoffs and colleagues, ${ }^{7}$ as well as other investigators, have made considerable contributions for ventricle models that serve as a foundation for many additional development and investigations. The in vivo MRI-based human $\mathrm{RV} / \mathrm{LV} / \mathrm{patch} / \mathrm{band}$ model adds the band to consideration as a potential surgical option for ventricle repair. Although the structure-only model was sufficient for our purposes in the present study, fluid-structure interaction models will be developed that will allow us to investigate both flow and structure stress/strain behaviors and their influence on various biologic processes, including ventricle remodeling and myocardium regeneration. As we continue our investigations of ventricular remodeling and myocardium tissue regeneration after stem cell seeding, detailed localized mechanical stress and flow conditions will be obtained to quantify their effects and influence on ventricular remodeling, myocardium tissue regeneration, and related cellular activities. Our RV/LV/patch fluid-structure interaction model can serve as a useful tool to investigate cellular biology and tissue regeneration under localized flow and structural stress environment.

\section{Model Limitations and Future Directions}

Several improvements can be added to our models in the future for better accuracy and applicability: a multiband design for better tricuspid valve closure to address the regurgitation issue; fluid-structure interaction to obtain both flow and structural stress/strain information for complete mechanical analysis; direct measurements of tissue mechanical properties for improved accuracy of our models; proper methods to model active contraction by adding active stress and techniques adjusting zero-stress sarcomere fiber length; inclusion of both tricuspid and pulmonary valve mechanics in the model to simulate regurgitation; and multiscale models, including organ, cell, and gene investigations. This will allow the mechanical conditions obtained from the organ level to pass to the microscopic level and cell activities obtained from the microscopic level to organ level to discover the mechanisms governing the tissue regeneration process. Diastolic dysfunction, defined as an abnormal stress-strain relationship when the heart is in a relaxed state, was not evaluated in the present model and will be investigated in the future using proper model assumptions and patient data for that purpose.

\section{CONCLUSIONS}

The CMR-based RV/LV/patch/band model introduced in the present report provides a proof of concept for using passive or actively contracting elastic bands to improve RV cardiac function. The effect of band material stiffness variations, band length, and active contraction were investigated. Our preliminary results indicate that band insertion, combined with active band contraction and tissue regeneration techniques that restore RV myocardium, has the potential to improve right ventricle EF by $7.5 \%$ and $4.2 \%$. The computational simulations suggested that additional investigations using in vitro experiments, animal models, and, finally, patient studies are warranted. The band insertion combined with myocardium regeneration techniques and $\mathrm{RV}$ remodeling surgical procedures has promising potential to improve ventricular function in patients with repaired TOF and other congenital heart diseases.

\section{References}

1. Therrien J, Siu SC, McLaughlin PR. Pulmonary valve replacement in adults late after repair of tetralogy of Fallot: are we operating too late? J Am Coll Cardiol. 2000;36:1670-5.

2. Vliegen HW, Van Straten A, De Roos A, Roest AA, Schoof PH, Zwinderman $\mathrm{AH}$, et al. Magnetic resonance imaging to assess the hemodynamic effects of pulmonary valve replacement in adults late after repair of tetralogy of Fallot. Circulation. 2002;106:1703-7.

3. Waien SA, Liu PP, Ross BL, Williams WG, Webb GD, McLaughlin PR. Serial follow-up of adults with repaired tetralogy of Fallot. J Am Coll Cardiol. 1992; 20:295-300.

4. Peskin CS. Numerical analysis of blood flow in the heart. J Com Phys. 1977;25: 220-52.

5. Peskin CS. A three-dimensional computational method for blood flow in the heart. J Comp Phys. 1989;81:372-405.

6. McCulloch AD, Waldman L, Rogers J, Guccione JM. Large-scale finite element analysis of the beating heart. Crit Rev Biomed Eng. 1992;20:427-49.

7. Kerckhoffs RC, Neal ML, Gu Q, Bassingthwaighte JB, Omens JH, McCulloch AD. Coupling of a 3D finite element model of cardiac ventricular mechanics to lumped systems models of the systemic and pulmonic circulation. Ann Biomed Eng. 2007;35:1-18

8. Hunter PJ, Pullan AJ, Smaill BH. Modeling total heart function. Annu Rev Biomed Eng. 2003;5:147-77.

9. Nash MP, Hunter PJ. Computational mechanics of the heart: from tissue structure to ventricular function. J Elast. 2000;61:113-41.

10. Costa KD, Takayama Y, McCulloch AD, Covell JW. Laminar fiber architecture and three-dimensional systolic mechanics in canine ventricular myocardium. Am J Physiol. 1999;276((2 Pt 2):H595-607.

11. Guccione JM, Waldman LK, McCulloch AD. Mechanics of active contraction in cardiac muscle: part II—cylindrical models of the systolic left ventricle. J Biomech Eng. 1993;115:82-90.

12. Axel L. Biomechanical dynamics of the heart with MRI. Annu Rev Biomed Eng. 2002; 4:321-47.

13. Saber NR, Gosman AD, Wood NB, Kilner PJ, Charrier CL, Firman DN. Computational flow modeling of the left ventricle based on in vivo MRI data: initial experience. Ann Biomech Eng. 2001;29:275-83.

14. Tang D, Yang C, Geva T, del Nido PJ. Image-based patient-specific ventricle models with fluid-structure interaction for cardiac function assessment and surgical design optimization. Prog Pediatr Cardiol. 2010;30: 51-62.

15. Tang D, Yang C, Geva T, Gaudette G, del Nido PJ. Multi-physics MRI-based two-layer fluid-structure interaction anisotropic models of human right and left ventricles with different patch materials: cardiac function assessment and mechanical stress analysis. Comput Struct. 2011;89:1059-68.

16. Tang D, Yang C, Geva T, del Nido PJ. Patient-specific MRI-based 3D FSI RV/LV/ patch models for pulmonary valve replacement surgery and patch optimization. J Biomech Eng. 2008;130:041010. 
17. del Nido PJ. Surgical management of right ventricular dysfunction late after repair of Tetralogy of Fallot: right ventricular remodeling surgery. Semin Thorac Cardiovasc Surg Pediatr Card Surg Annu. 2006;9:29-34.

18. Geva T, Gauvreau K, Powell AJ, et al. Randomized trial of pulmonary valve replacement with and without right ventricular remodeling surgery. Circulation. 2010;122(11 Suppl):S201-8.

19. Bathe KJ. Finite element procedures. Upper Saddle River, NJ: Prentice Hall; 1996.

20. Sacks MS, Chuong CJ. Biaxial mechanical properties of passive right ventricular free wall myocardium. J Biomech Eng. 1993;115:202-5.

21. Humphrey JD. Cardiovascular Solid Mechanics. New York: Springer-Verlag; 2002.

22. Holzapfel GA, Gasser TC, Ogden RW. A new constitutive framework for arterial wall mechanics and a comparative study of material models. J Elasticity. 2000; 61:1-48.

23. Sanchez-Quintana D, Anderson R, Ho SY. Ventricular myoarchitecture in tetralogy of Fallot. Heart. 1996;76:280-6.
24. Kochupura PV, Azeloglu EU, Kelly DJ, et al. Tissue-engineered myocardia patch derived from extracellular matrix provides regional mechanical function Circulation. 2005;112(9 Suppl):I144-9.

25. Kelly DJ, Rosen AB, Schuldt AJ, et al. Increased myocyte content and mechanical function within a tissue-engineered myocardial patch following implantation. Tissue Eng Part A. 2009;15:2189-201.

26. Proulx MK, Carey SP, Ditroia LM, et al. Fibrin microthreads support mesenchymal stem cell growth while maintaining differentiation potential. J Biomed Mater Res A. 2011;96:301-12.

27. Aleksova A, Masson S, Maggioni AP, et al; on the behalf of the CandHeart Investigators. Effects of candesartan on left ventricular function, aldosterone and BNP in chronic heart failure. Cardiovasc Drugs Ther. February 2, 2012 [Epub ahead of print].

28. Grossi EA, Woo YJ, Schwartz CF, et al. Comparison of Coapsys annuloplasty and internal reduction mitral annuloplasty in the randomized treatment of functional ischemic mitral regurgitation: impact on the left ventricle. $J$ Thorac Cardiovasc Surg. 2006;131:1095-8. 
Stress-stretch curves for patch, scar andRV tissue

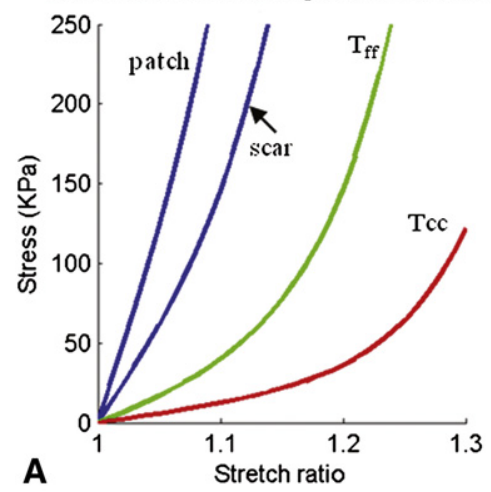

Imposed RV inner pressure matching CMR data

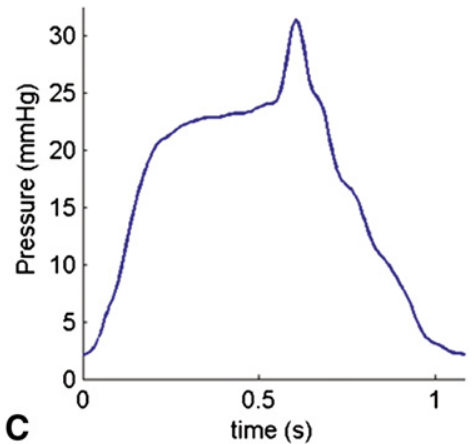

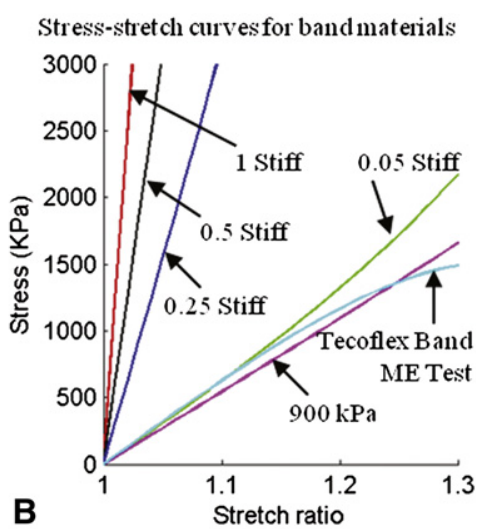

Computational $\mathrm{RV}$ volume matching CMR-measured $R V$ volume data.

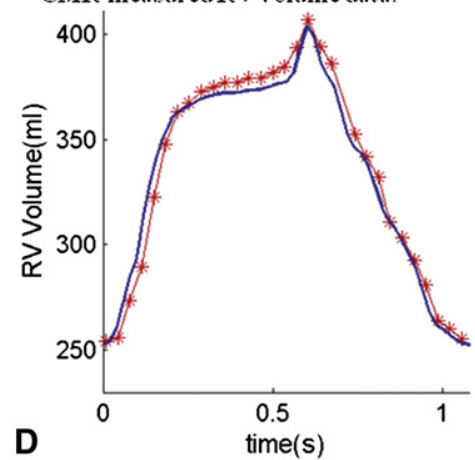

FIGURE E1. Material stress-stretch curves, pressure conditions used in report and computational right ventricular $(R V)$ volume curve matching cardiac magnetic resonance (CMR)-measured data. A, Stress-stretch curves from Mooney-Rivlin isotropic patch, scar, and anisotropic RV tissue models. Model parameter values $\left(\mathrm{c}_{2}=0\right.$ for all models). Anisotropic RV model (out-layer given in plot): $\mathrm{c}_{1}=5.746 \mathrm{kPa}, \mathrm{c}_{2}=0, \mathrm{D}_{1}=1.479 \mathrm{kPa}, \mathrm{D}_{2}=3.0$, $\mathrm{K}_{1}=23.413 \mathrm{kPa}, \mathrm{K}_{2}=3.2 ; \mathrm{scar}, \mathrm{c}_{1}=19.227 \mathrm{kPa}, \mathrm{c}_{2}=0, \mathrm{D}_{1}=19.227 \mathrm{kPa}, \mathrm{D}_{2}=9.0$; patch, $\mathrm{c}_{1}=38.454 \mathrm{KPa}, \mathrm{c}_{2}=0, \mathrm{D}_{1}=38.454 \mathrm{kPa}, \mathrm{D}_{2}=9.0$. $T_{f f}$, stress in the fiber direction; $T c c$, stress in fiber circumferential direction. B, Stress-stretch curves from Mooney-Rivlin isotropic models for passive band materials. 1 stiff, $\mathrm{c}_{1}=12,000 \mathrm{kPa}, \mathrm{D}_{1}=6000 \mathrm{kPa}, \mathrm{D}_{2}=1.4 ; 0.5$ stiff: $\mathrm{c}_{1}=6000 \mathrm{kPa}, \mathrm{D}_{1}=3000 \mathrm{kPa}, \mathrm{D}_{2}=1.4 ; 0.25$ stiff, $\mathrm{c}_{1}=3000 \mathrm{kPa}$, $\mathrm{D}_{1}=1500 \mathrm{kPa}, \mathrm{D}_{2}=1.4 ; 0.05$ stiff, $\mathrm{c}_{1}=600 \mathrm{kPa}, \mathrm{D}_{1}=300 \mathrm{kPa}, \mathrm{D}_{2}=1.4$; curve fitting mechanical engineering testing data, $\mathrm{c}_{1}=1510 \mathrm{kPa}$, $\mathrm{D}_{1}=-221.9 \mathrm{kPa}, \mathrm{D}_{2}=2.0 ; 900 \mathrm{kPa}$ band, $\mathrm{c}_{1}=900 \mathrm{kPa}, \mathrm{D}_{1}=0 \mathrm{kPa}, \mathrm{D}_{2}=0$. C, Imposed inner RV pressure. $\mathrm{D}$, Model validation, computational RV volume from baseline no-band model matching CMR-measured RV volume data. 

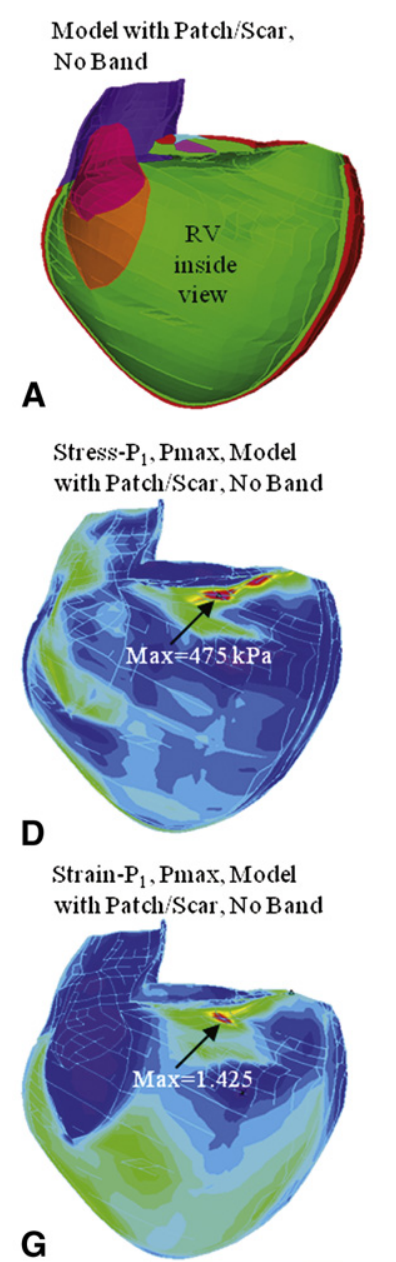

(Min

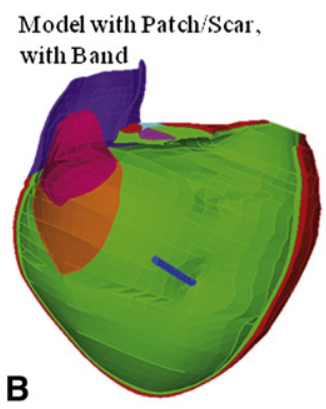

Stress- $\mathrm{P}_{1}$, Pmax, Model with $\mathrm{Patch} / \mathrm{Scar}$ and $\mathrm{B}$ and

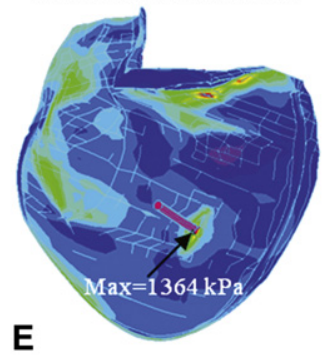

Strain- $\mathrm{P}_{1}, \mathrm{Pmax}$, Model with $\mathrm{Patch} / \mathrm{Scar}$ and $\mathrm{B}$ and

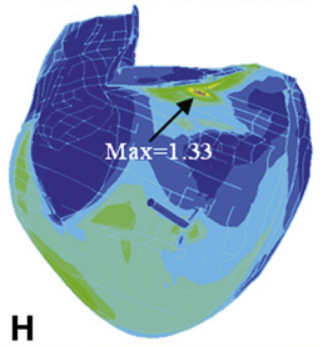

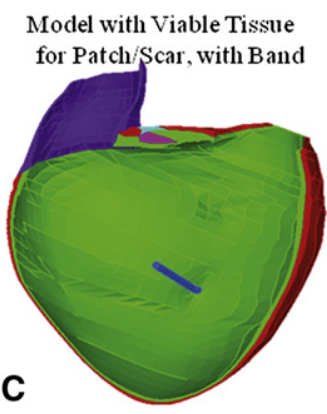

Stress- $\mathrm{P}_{1}, \mathrm{Pmax}, \mathrm{w} / \mathrm{B}$ and, viable tissue for $\mathrm{patch} / \mathrm{Scar}$

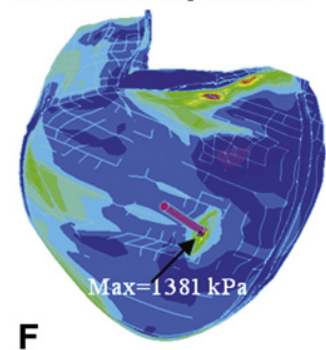

Strain- $\mathrm{P}_{1}, \mathrm{Pmax}, \mathrm{w} / \mathrm{B}$ and, viable tissue for $p$ atch $/ \mathrm{Scar}$

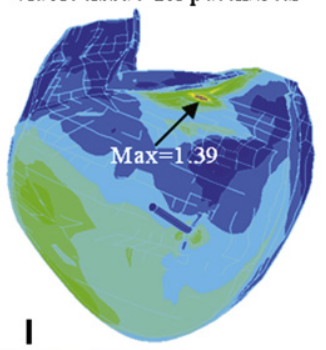

FIGURE E2. Stress and strain plots from 3 models corresponding to maximum pressure condition showing complex stress/strain behaviors. A, Model with patch and scan, no band. B, Model with patch and scan, with band. C, Model with viable tissue for patch and scan, with band. D, Stress- ${ }_{1}$, Pmax, model with patch and scan, no band. E, Stress- $\mathrm{P}_{1}$, Pmax, model with patch, scar, and band. F, Stress- $\mathrm{P}_{1}$, Pmax, model with viable tissue for patch and scan, with band. G, Strain- $\mathrm{P}_{1}$, Pmax, model with patch and scan, no band. H, Strain- $\mathrm{P}_{1}$, Pmax, model with patch, scar, and band. I, Strain- $\mathrm{P}_{1}$, Pmax, model with viable tissue for patch and scan, with band. 